\title{
The CSIRO Total Wellbeing Diet Book 1: sociodemographic differences and impact on weight loss and well-being in Australia
}

\author{
Belinda Wyld*, Adam Harrison and Manny Noakes \\ Commonwealth Scientific Industrial Research Organization, Food and Nutritional Sciences, PO Box 10041, \\ Adelaide BC, South Australia, Australia
}

Submitted 29 October 2009: Accepted 23 February 2010: First published online 15 April 2010

\begin{abstract}
Objective: The CSIRO Total Wellbeing Diet (TWD) publication is an evidencebased weight management strategy utilising a structured higher protein diet as part of a nutritionally balanced lifestyle programme. Despite its popularity, the impact of TWD on weight status, weight loss and food choices of Australians was unknown.

Design: An independent representative survey was conducted in 2006. Sociodemographic differences in awareness, use of TWD and the impact on weight status and well-being were investigated via computer-aided telephone interviews and web-based surveys.

Setting: Australia.

Subjects: A total of 5026 men and women aged 18-60 years.

Results: Consumers were highly aware of TWD (66\%) with personal use reported by $7.5 \%$ of the total sample ( $n$ 5026). An additional $2.5 \%$ (126 people) were members of a household that used TWD. In all, $80 \%$ of TWD purchasers actively used the eating plan with approximately $3 \cdot 8 \%$ losing an average self-reported weight loss of $5.7 \mathrm{~kg}$ ( $\mathrm{SD}=1.72 \mathrm{~kg}$; range $=1-13 \mathrm{~kg}$ ). Results showed that awareness was greatest among women $(73 \cdot 79 \% v .58 \cdot 27 \%)$, those over 50 years of age $(69 \cdot 39 \% v \cdot 62 \cdot 88 \%)$ with no children in the household $(69 \cdot 00 \% v$. $64 \cdot 88 \%)$, tertiary educated people $(72 \cdot 58 \% v .63 \cdot 22 \%)$ and those with more previous weight loss attempts $(79 \cdot 66 \% v .70 \cdot 24 \%)$. Logistic regression was unable to predict an identifiable sociodemographic profile of TWD users.

Conclusions: The present study shows widespread uptake of TWD in Australia with few sociodemographic differences. Self-reported increased awareness of nutrition and well-being as well as weight loss indicates that TWD has been a successful delivery mechanism for lifestyle advice.
\end{abstract}

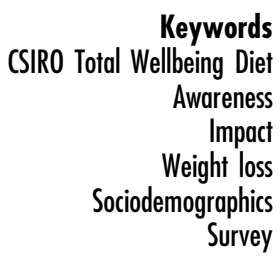

Overweight and obesity is a key public health challenge for Australia. In 2007-2008, self-reported data from the National Health Survey showed that over half of Australian men and women were classified as being overweight or obese ${ }^{(1)}$. It has been estimated that on average, women in 1999 weighed $4.8 \mathrm{~kg}$ more than their counterparts in 1980 and men $3.6 \mathrm{~kg}$ more ${ }^{(2)}$, with energy intakes increasing by around $350 \mathrm{~kJ} / \mathrm{d}$ for adults between 1983 and $1995^{(3)}$. In addition, the cost of obesity (not including overweight) in Australia has been estimated by Access Economics in 2008 to be $\$ 58.2$ billion - more than five times the cost of Medicare ${ }^{(4)}$ - with a significant portion of this cost attributed to lost well-being and chronic medical conditions associated with overweight and obesity ${ }^{(5,6)}$.

The Commonwealth Scientific Industrial Research Organisation (CSIRO) has historically been a major source of nutritional research on food and dietary patterns for metabolic health in Australia. The CSIRO Total Wellbeing Diet (TWD) ${ }^{(7)}$ was developed following several shortterm and long-term clinical trials by CSIRO and international researchers showing beneficial effects of higher protein patterns in weight loss and body composition $^{(8-11)}$. TWD is a structured, nutritionally balanced eating plan for weight control. Launched in 2004, the TWD commercial publication presented the scientific basis behind the diet, as well as a template meal plan, 12 weeks of sample menus, shopping lists, mainstream recipes and a structured exercise guide. At the time of writing, TWD had sold over 1 million copies in Australia alone, with seventeen foreign language translations and revised English editions in the United States, the United Kingdom, Canada and South Africa.

The popularity of TWD suggested a high level of public receptiveness to communication on healthy lifestyles. However, the impact or otherwise of TWD on weight 
status, weight loss and food choices of Australians was unknown. As a result, a study was independently conducted to evaluate awareness of TWD, how it was being used in the community and whether there was a sociodemographic profile of people who achieved successful weight control and/or maintenance using TWD.

\section{Experimental methods}

Inside Story, a Sydney-based research company, was commissioned to carry out a nationally representative survey assessing awareness of TWD, and its impact on weight status and well-being. The survey was conducted in September 2006, before the launch of TWD Book $2^{(12)}$. A multi-modal approach was employed consisting of two separate arms: (i) a 10 min computer-aided telephone interview (CATI) and (ii) a $15 \mathrm{~min}$ web-based survey. Given the increase in home Internet access in Australia from $16 \%$ in 1998 to $64 \%$ in 2006-2007 for all age groups $^{(13)}$, this combined approach to data collection was considered to be a robust way of capturing a broad crosssection of the Australian community.

The participant pool for the CATI was generated from Random Digit Dialling methodology. Once the household had been contacted, researchers requested to speak to a person in the household over 18 years of age who had the most recent birthday. As per standard market research practice, the CATI was performed on a quota basis and data weights were further applied to individual cases for gender, age, state and region (metropolitan or regional) to align with Australian 2006 census data ${ }^{(13)}$. Participants for the web-based surveys were drawn from a research panel where they had opted in to partake in marketing and research activities. The sample was not paid to complete the survey and all Internet surveys were statistically weighted by gender, age and location to align with the Australian population.

Data from both arms of the study were a combination of forced and open responses with the length of the interview depending on participant responses to their awareness and use of TWD. Analysis was performed using $\chi^{2}$-tests, effect size statistics relevant to the variables $(\Phi$, Cramer's $V$ or Somers' $d$ ) and logistic regression. All analyses were performed using the Statistical Package for the Social Sciences statistical software package version $14 \cdot 0$ (SPSS Inc., Chicago, IL, USA).

\section{Results}

\section{Sample}

The sample consisted of 2513 men and 2513 women from across regional and metropolitan areas of Australia. Participants ranged from 18 years of age to over 60 years of age and responded to only one arm of the study (Table 1 ). The present study targeted a general population-representative
Table 1 Number of participants by region breakdown by study arm

\begin{tabular}{lccc}
\hline & $\begin{array}{c}\text { Telephone (10 min) } \\
(n \text { 1000) }\end{array}$ & $\begin{array}{c}\text { Online (15 min }) \\
(n \text { 4026) }\end{array}$ & $\begin{array}{c}\text { Total } \\
(n \text { 5026) }\end{array}$ \\
\hline Metropolitan & 702 & 2824 & 3526 \\
Regional & 298 & 1202 & 1500 \\
Total & 1000 & 4026 & 5026 \\
\hline
\end{tabular}

sample of Australians with respect to age, proportion of men and women and region. Different socio-economic status (SES) groups were not specifically targeted, nor excluded, from the study. As our sample had a somewhat higher proportion of respondents who were in or attached to the labour force $(80 \cdot 0 \% v$. 64.6\%), and educated to a tertiary or post-graduate level $(32 \cdot 3 \% v$. $23.0 \%$ ), the following results are representative of middleclass working Australians ${ }^{(14)}$.

\section{Awareness of TWD}

Over two-thirds $(66 \cdot 18 \%)$ or 3326 people indicated an awareness of the CSIRO eating plan. Although awareness across all age and gender categories exceeded 50\%, total awareness was greatest in women and those aged over 50 years. When looking at the available sources of awareness, traditional media such as television, radio and print mediums had the strongest impact on the total sample (2262/5026 people). Word of mouth was also effective with 698 of $5026(13.89 \%)$ people reporting that they first heard about TWD through friends/family/colleagues. Of those aware of TWD (3326 of 5026 people), over $85 \%$ reported that their impressions of the dietary plan was either a long-term eating and lifestyle pattern for weight loss or intended for overall health and well-being.

\section{TWD uptake and usage}

Of those aware of TWD, 10\% had purchased a copy of the book mainly for themselves (69\%) or their household (28\%). At the time of the present study, there were $7 \cdot 6$ million households in Australia, suggesting that approximately 760000 households had purchased TWD. Despite the fact that these figures may be inflated owing to multiple purchases from the same household, this does match sales records from 2006, which indicated that between 700000 and 800000 copies had been sold in Australia (S. Symonds, Nielson BookScan, oral communication, 3 February 2010 and L. Cullen, Penguin Group, Australia, private communication, 2 February 2010).

The main reason for purchasing TWD was to help lose weight $(47 \cdot 3 \%$ and $37 \cdot 8 \%)$ and to improve their diet and eat healthier $(27 \cdot 0 \%$ and $30 \cdot 8 \%$ ). In all, $5 \cdot 0 \%$ (seventeen people) were recommended TWD by their doctor to manage a specific medical condition(s), whereas a further $22 \cdot 8 \%$ (eighty people) were recommended the book by a friend.

A total of 504 of 5026 participants reported that TWD was used in their household either personally (378 people) or by their partner/housemate (126 people). In all, 
Table 2 Differences in TWD awareness across dichotomous sociodemographic categories

\begin{tabular}{|c|c|c|c|c|}
\hline & \multicolumn{2}{|c|}{ Heard of TWD } & \multicolumn{2}{|c|}{ Have not heard of TWD } \\
\hline & $n$ & $\%$ & $n$ & $\%$ \\
\hline \multicolumn{5}{|l|}{ Gender } \\
\hline Male & 1437 & $58 \cdot 27$ & 1029 & $41 \cdot 73$ \\
\hline Female & 1889 & $73 \cdot 79$ & 671 & $26 \cdot 21$ \\
\hline \multicolumn{5}{|l|}{ Age (years) } \\
\hline $18-49$ & 1262 & $62 \cdot 88$ & 745 & $37 \cdot 12$ \\
\hline$\geq 50$ & 1920 & $69 \cdot 39$ & 847 & $30 \cdot 61$ \\
\hline \multicolumn{5}{|l|}{ Household structure } \\
\hline Children in the household & 1413 & $64 \cdot 88$ & 765 & $35 \cdot 12$ \\
\hline No children in the household & 1504 & $69 \cdot 00$ & 676 & $31 \cdot 00$ \\
\hline \multicolumn{5}{|l|}{ Education } \\
\hline Tertiary & 1199 & $72 \cdot 58$ & 453 & $27 \cdot 42$ \\
\hline Non-tertiary & 2095 & $63 \cdot 22$ & 1219 & $36 \cdot 78$ \\
\hline \multicolumn{5}{|l|}{ Weight loss attempts } \\
\hline Less than five attempts & 1362 & $70 \cdot 24$ & 577 & $29 \cdot 76$ \\
\hline Five or more attempts & 987 & $79 \cdot 66$ & 252 & $20 \cdot 34$ \\
\hline
\end{tabular}

TWD, Total Wellbeing Diet.

$80 \%$ of TWD purchasers actively used the eating plan. The main reason reported by respondents for buying and not subsequently using TWD was procrastination - they meant to use TWD, but had yet to get around to it (56\%). On the basis of the population figures for Australians aged between 15 and 64 years, the projected figures are $10 \%$ of Australians (approximately 1.3 million people) lived in a household that used TWD in some way.

Of the $2.5 \%$ of people who reported either loosely or strictly following the diet, eighty-eight people provided information on whether they were still following the menu plans: $6 \cdot 8 \%$ were still carefully following the plan, $28 \cdot 4 \%$ were still loosely following the plans, $19 \cdot 3 \%$ were using the plans for guidance and $45 \cdot 5 \%$ were no longer following the plans.

\section{Sociodemographic differences in TWD awareness}

Additional analyses were performed to examine any differences in sociodemographic characteristics among those aware of TWD. The variables available for use included education, work status, household structure, attitude to food/diet, present weight loss attempts, previous weight loss attempts, gender, age group, region and state.

As $\chi^{2}$ analyses indicated only weak sociodemographic differences between categories of positive awareness, a general 'heard of TWD' category was created. Some of the multi-level dependent variables were also reduced to dichotomous to allow clearer analysis and interpretation of the data: education ('tertiary educated' and 'non-tertiary educated'), household structure ('children in the household' and 'no children in the household'), previous weight loss attempts (median split of 'under five attempts' and 'five or more attempts'), and age (median split of '18-49 years' and '50 years or older').

The demographic split of respondents across awareness is presented in Table 2 . Results showed that men $\left(\chi^{2}(1\right.$, $n=5026)=134 \cdot 41, \quad P=0 \cdot 000)$ and those people with children present in the household $\left(\chi^{2}(1, n=4358)=8 \cdot 15\right.$, $P<0.05)$ had less awareness of TWD. However, those people most likely to be aware of TWD were 50 years of age or older $\left(\chi^{2}(1, n=4774)=21 \cdot 88, P=0 \cdot 000\right)$, tertiary educated $\left(\chi^{2}(1, n=4966)=42 \cdot 85, P=0 \cdot 000\right)$ and had reported more previous weight loss attempts $\left(\chi^{2}(1\right.$, $n=3178)=34 \cdot 29, P=0 \cdot 000)$.

\section{Sociodemographic differences in TWD uptake and usage}

Despite TWD being developed as a structured eating and exercise pattern for weight loss, the publication appeared to be used in multiple ways in the community (Table 3 ). In order to assess this, usage type was divided into two distinct categories: (i) usage that should have a direct impact on weight/health ('health': $n$ 261, 76.5\%); and (ii) usage that may have an indirect impact on weight/health through changing food culture ('culture': $n$ 80, 23.5\%). Sociodemographic variables were then examined to determine whether these could help explain why participants may have used TWD in different ways. Results showed that having children in the house was weakly related to a smaller proportion of people using TWD in a direct, health-related way $\left(\chi^{2}(1, n=298)=6 \cdot 21\right.$, $P<0 \cdot 05, \Phi=0 \cdot 152, \quad P<0 \cdot 01)$. However, the reduced variables of tertiary education and previous weight loss were non-significant.

\section{Sociodemographic profile of TWD users}

A further aim of the present study was to determine whether there was a sociodemographic profile that was characteristic of TWD users. A logistic regression was performed to assess the usefulness of the measured sociodemographic variables in predicting whether someone had used TWD. Despite the regression model passing the goodness-of-fit criteria with statistical significance $\left(\chi^{2}=70 \cdot 114,(27), P=0 \cdot 000\right)$, the variables 
Table 3 Ways in which the CSIRO Total Wellbeing Diet was being used ${ }^{\star}$

\begin{tabular}{lrr}
\hline & $n$ & \multicolumn{1}{c}{${ }^{-}$} \\
\hline Used the menu plan & 166 & 207 \\
Used the basic principles and adapted them to my lifestyle & 262 & $56 \cdot 4$ \\
Used the recipes and meal ideas & 75 & $71 \cdot 6$ \\
Read it but have not yet put specific ideas into practice & 182 & $20 \cdot 5$ \\
Used it for general nutritional information & $49 \cdot 7$ \\
\hline
\end{tabular}

CSIRO, Commonwealth Scientific Industrial Research Organisation.

${ }^{*}$ As multiple responses were possible, this adds up to more than $100 \%$ and $n 366$.

explained extremely small amounts of the variance (Cox and Snell $R^{2}=0 \cdot 052$, Nagelkerke $R^{2}=0 \cdot 079$ ). The final model was only able to accurately predict TWD usage in four cases, indicating that no clearly identifiable profile for TWD users could be established. This suggests that TWD was used by a wide cross-section of the population.

\section{Impact on weight loss and well-being}

Self-reported weight loss data were available for 501 of 503 respondents who reported using TWD in their household. Data suggested that $3 \cdot 8 \%$ of the total sample lost weight while using TWD. Among these, 0.66\% (thirty-three participants) reported losing weight as a result of another household member using TWD. When these figures are projected to the Australian population, they indicate that approximately $4 \%$ of Australians aged between 18 and 64 years of age (547200 people) may have lost weight on TWD. This matches sales figures for 2006, which showed an average number of TWD publications sold to be between 700000 and 800000 (S. Symonds, Nielson BookScan, oral communication, 3 February 2010 and L. Cullen, Penguin Group, Australia, private communication, 2 February 2010).

The average amount of weight loss reported was $5 \cdot 7$ (sD 1.74 ) $\mathrm{kg}$ (range: $1-13 \mathrm{~kg}$ ). Approximately $30 \%$ of the sample reported a weight maintenance period in excess of 6 months, with a further $33 \%$ indicating that they had maintained their weight for 3-6 months. The amount of weight loss was moderately correlated with the length of weight maintenance $(r=0 \cdot 40, P<0 \cdot 001)$, suggesting that those participants who lost more weight also maintained this weight loss for longer.

The impact of TWD can also be measured by its effect on psychosocial variables. Of those participants who had used TWD, a majority reported improvements in the following areas of their life (open multiple choice):

- attitude to health and well-being (71\%);

- overall health (63\%);

- energy levels (61\%);

- level of concern they have about their health (57\%); and

- overall fitness (57\%).

In addition, changes in relation to food and eating behaviours were also expressed, with participants who had used TWD in their households reporting that they (open multiple choice):

- are more conscious of eating healthy foods $(57 \cdot 3 \%)$;

- cook more at home (42.3\%);

- eat more fish (44.4\%);

- drink more water $(55 \cdot 6 \%)$;

- eat less junk food (49.2\%);

- eat more vegetables $(52 \cdot 2 \%)$; and

- eat more fruit (43.3\%).

The use of TWD among participants also had tangible health benefits, with reported self-diagnosed improvements in blood pressure (25\% or 130 participants), glucose levels ( $23 \%$ or 116 participants) and cholesterol levels ( $29 \%$ or 148 participants).

\section{Discussion}

The CSIRO TWD appears to have had a significant impact on self-reported health and well-being of consumers. In all, $66 \%$ of Australian consumers surveyed were aware of TWD, with approximately $10 \%$ of Australians having lived in a household that used TWD in some way. A projected $4 \%$ of the population has lost weight as a result of TWD, with an average weight loss of $5.7 \mathrm{~kg}$. In addition, TWD appears to have been taken up in a variety of ways across wide sections of the community with the use of recipes, menu plans and nutrition information being key elements of usage patterns.

With overweight and obesity strongly linked with significant medical problems such as CVD, type 2 diabetes, some cancers and osteoarthritis, it is interesting to note that $5 \%$ of respondents were recommended the publication by their general practitioner to manage a specific medical condition(s). Furthermore, information from users showed that they believed TWD had physiological health benefits in assisting them to improve their blood pressure, glucose and cholesterol levels.

Analyses across sociodemographic categories suggested broad-based TWD uptake and usage in the mainstream Australian population.

Scientific evaluations of the impact diet book publications have on health and psychosocial outcomes of consumers are scarce. However, the media support for 
TWD and the associated social marketing campaign makes it comparable to other Australian government health campaigns, such as the Go for $2 \& 5^{\circledR}$ health initiative and the Measure Up campaign. Although the Measure Up initiative has not been evaluated to date, evaluations of the Go for $2 \& 5^{\circledR}$ campaign have been conducted both nationally and in Queensland and Western Australia ${ }^{(15,16)}$. The National survey $^{(15)}$ was conducted over 3 months in 2005 via a series of pre- and post-campaign telephone surveys. The Perthbased evaluation ${ }^{(16)}$ however, tracked fruit and vegetable attitudes, beliefs and consumption continuously over 3 years from 2002 to 2005 via two independent telephone surveys. Results of the Queensland evaluation have not been published. Both campaign evaluations showed a significant increase in consumer awareness of the recommended intakes for fruits and vegetables. However, although behavioural changes in consumption were only slight from pre- and post-test in the national evaluation, the Western Australian study showed stronger increases in the mean number of daily fruit and vegetable servings throughout the 3-year marketing campaign. The principles of TWD are consistent with the Go for $2 \& 5^{\circledR}$ health initiative and recommend the consumption of two servings of fruit and two and a half cups of vegetables per day. In line with the above evaluations, participants in the current investigation also reported eating more fruits and vegetables as a result of using the dietary plan. However, of more interest is that both the Go for $2 \& 5^{\circledR}$ campaign and the current study have highlighted the importance of assessing the impact of such initiatives in supporting cultural changes in food and behaviour. To our knowledge, this is the first Australian-wide population evaluation to consider weight loss and other health changes (although self-reported) as well as dietary and behavioural changes as a result of a commercial publication.

The present study has shown that healthy lifestyle information produced by a credible organisation through a popular commercial media, such as book publications, may be successful in motivating changes in eating and exercise behaviours and weight status.

\section{Study limitations}

Although the present study was representative of the broad general Australian population in terms of age, gender and region, it did not have the scope to assess the access, use and success of TWD among specific SES groups. Both this eating pattern and healthier eating patterns in general may pose greater difficulties for those less financially secure ${ }^{(17,18)}$. Differences in education level in the present study may be due to self-selection and the perceived salience of health concerns; however, it is important to acknowledge that TWD may not be relevant nor appropriate for Australians from lower educational and financially less secure backgrounds. Implementing healthy lifestyle programmes for these target groups is necessary, yet challenging, and mainstream middle-class programmes such as TWD may not be the most effective way of delivering and educating socially disadvantaged groups about the values and benefits of a healthy lifestyle.

Furthermore, the present study relied on self-report methodology to ascertain impacts of TWD on weight, which could have produced a response bias. Although evaluations of other healthy eating campaigns, such as the Go for $2 \& 5^{\circledR}$ campaign, also use self-report for data collection, they have often employed a pre-and-post-test intervention design which is stronger than the observational post-test design employed in the present study.

As the current investigation did not collect data that were able to capture those Australian consumers who potentially regained weight after ceasing TWD, it has not provided an in-depth understanding of the barriers and facilitators to success and maintenance of TWD. An evaluation of this information could have enabled targeted programme development that would maximise long-term weight loss. However, it does indicate that procrastination is an expressed barrier that may have implications for interventions that aim to shift the community along the behavioural change continuum.

\section{Acknowledgements}

The present study received no specific grant from any funding agency in the public, commercial, or not-forprofit sectors. The authors have no conflict of interest, B.W. was responsible for the study, coordination and analysis and preparation of the manuscript. A.H. assisted in the analysis and manuscript write-up. M.N. was responsible for assisting in the study design and overall supervision of the project.

\section{References}

1. Australian Bureau of Statistics (2009) National Health Survey: summary of results 2007-08; catalogue number 4364.0. http://www.abs.gov.au/ausstats/abs@.nsf/mf/4364.0/ (accessed August 2009).

2. Australian Bureau of Statistics (1995) How Australians measure up. http://www.ausstats.abs.gov.au/Ausstats/ subscriber.nsf/Lookup/CA25687100069892CA256889001F4A36/ \$File/43590_1995.pdf (accessed October 1998).

3. Cook T, Rutishauser I \& Allsop R (2001) The Bridging Study - Comparing Results from the 1983, 1985 and 1995 Australian National Nutrition Surveys. Canberra: Australian Food and Nutrition Monitoring Unit, Commonwealth Department of Health and Aged Care.

4. Access Economics (2008) The Growing Cost of Obesity in 2008: Three Years on. Canberra: Diabetes Australia.

5. O'Dea JA (2008) Gender, ethnicity, culture and social class influences on childhood obesity among Australian schoolchildren: implications for treatment, prevention and community education. Health Soc Care Community 16, 282-290.

6. Mathers C, Vos T \& Stevenson C (1999) The Burden of Disease and Injury in Australia. Catalogue no. PHE17. Canberra: AIHW. 
7. Noakes M \& Clifton P (2005) The CSIRO Total Wellbeing Diet. Australia: Penguin Books.

8. Farnsworth E, Luscombe ND, Noakes M et al. (2003) Effect of a high-protein, energy-restricted diet on body composition, glycemic control, and lipid concentrations in overweight and obese hyperinsulinemic men and women. $A m J$ Clin Nutr 78, 31-39.

9. Luscombe ND, Clifton PM, Noakes M et al. (2003) Effect of a high-protein, energy-restricted diet on weight loss and energy expenditure after weight stabilization in hyperinsulinemic subjects. Int J Obes 27, 582-590.

10. Noakes M, Keogh JB, Foster PR et al. (2005) Effect of an energy-restricted, high-protein, low-fat diet relative to a conventional high-carbohydrate, low-fat diet on weight loss, body composition, nutritional status, and markers of cardiovascular health in obese women. Am J Clin Nutr 81, 1298-1306.

11. Clifton PM, Keogh JB \& Noakes M (2008) Long-term effects of a high-protein weight-loss diet. Am J Clin Nutr 87, 23-29.

12. Noakes M \& Clifton PM (2006) The CSIRO Total Wellbeing Diet Book 2. Australia: Penguin Books.
13. Australian Bureau of Statistics (2006) Census of population and housing. http://www.abs.gov.au/websitedbs/d3310114. nsf/Home/census (accessed January 2007).

14. Australian Bureau of Statistics (2008) Australian social trends; catalogue number 4102.0. http://www.ausstats.abs. gov.au/Ausstats/subscriber.nsf/0/DE5DE30C9CF6E5E3CA 25748E00126A25/\$File/41020_2008.pdf (accessed January 2010).

15. Woolcock Research (2007) Research report: evaluation of the National Go for $2 \& 5^{\circledR}$ campaign. http://www.health. gov.au/internet/healthyactive/publishing.nsf/Content/2\&5eval-jan07 (accessed May 2009).

16. Pollard CM, Miller MR, Daly AM et al. (2008) Increasing fruit and vegetable consumption: success of the Western Australian go for $2 \& 5^{\circledR}$ campaign. Public Health Nutr 11, 314-320.

17. Brimblecombe JK \& O'Dea K (2009) The role of energy cost in food choices for an Aboriginal population in Northern Australia. Med J Aust 190, 549-551.

18. Kettings C, Sinclair AJ \& Voevodin M (2009) A healthy diet consistent with Australian health recommendations is too expensive for welfare-dependent families. Aust N Z J Public Health 33, 566-572. 